\title{
Shared Urges? The Links Between Drugs of Abuse, Eating, and Body Weight
}

\author{
Laurence J. Nolan
}

Published online: 10 February 2013

(C) Springer Science+Business Media New York 2013

\begin{abstract}
The results of several behavioral and neurophysiological studies suggest that some obese people respond to foods in a manner that resembles the behavior of drug dependent persons toward drugs. Indeed, there are common mechanisms in the brain responsible for the reinforcement of eating and drug use by food and drugs respectively and for the cue incentives associated with them. There is some evidence that dependence in one modality may influence the other. Active drug use is associated with normal (or even low) body weight but recovery from drug dependence may lead to increased consumption of food and ultimately to weight gain. It is possible that drug abstinence leads to incentive seeking in the food domain, increased food consumption, and weight gain. Thus, those in treatment for substance use disorders may benefit from weight maintenance counseling.
\end{abstract}

Keywords Substance dependence · Drugs - Abuse · Reward · Obesity $\cdot$ Eating behavior $\cdot$ Addiction . Incentive motivation $\cdot$ Body weight

\section{Introduction}

There are significant links between food consumption and drug use. Commonalities in the response to food of some obese persons and to drugs of drug dependent persons has led increasingly to the suggestion that food addiction may, in part, explain the recent dramatic increases in obesity in much of the world. Since 2008, the number of published scientific papers indexed in Medline with food addiction as a key term has increased sevenfold [1]. Much of the

\section{J. Nolan $(\bowtie)$}

Department of Psychology, Wagner College, 1 Campus Rd., Staten Island, NY 10301, USA

e-mail: LNolan@wagner.edu evidence provided to support this proposition comes from both behavioral and neurophysiological data. In brainimaging studies, striatal dopamine function in the obese and drug dependent show similar dysfunction in response to foods and drugs and, more importantly, the cues associated with them [2•]. These responses occur in concert with lack of inhibition: prefrontal cortex impulse control inputs are reduced [2•]. Other areas of brain dysfunction in both the obese and drug dependent include systems (i.e., the habenula, a structure near the pineal gland) involved in signaling of when reward fails to materialize (associated with relapse in the drug dependent). Furthermore, there is overlap of areas (i.e., insula) involved in drug craving and gustation [2•]. In animals, the existence of cross-sensitization from sugar to drugs and other behavioral parallels are also supportive of these links [3]. In the clinical arena, similarities between binge eating and substance use disorders (SUD) may be useful in attempting to understand the rise in obesity (in particular: escalation of use, loss of control, social consequences, and personal distress dimensions) [4]. However, despite some similarities, food addiction may not meet the criteria currently used to diagnose SUD, especially in regard to tolerance, displacement of important social and occupational activities by eating, and application of eating-related impairment thresholds among others [5•].

The food addiction literature has borrowed significantly from the drug addiction literature in part due to evidence of common neural substrates for food and drug reinforcement. Indeed, numerous studies of persons with SUD suggest a link between drug and alcohol use and food preference and consumption. Models of food addiction suggest that since neural mechanisms and cue incentives for drugs and foods are similar, perhaps there is an interaction between compulsive food and drug use. An important question that remains is whether drug use affects food consumption (or vice versa) and body weight. Are those who use drugs of abuse at 
higher risk for obesity? The purpose of this paper is to examine evidence that there is an association between eating behavior, body weight, and drug use in humans.

\section{Substance Use and Eating Disorders}

Recent reviews indicate that SUD has high co-occurrence with those eating disorders (ED) that include bulimic behaviors $[6,7]$. The majority of studies describing co-occurrence report ED as primary and SUD as secondary which may reflect the interest of ED researchers but also may reflect use by ED patients of stimulants to suppress appetite [7]. Most of the research in this area examines only the relationship between co-occurrence of diagnostic categories but ED is also associated with elevated frequency of drug use in those without SUD. Interestingly, this elevation was not in stimulant use (used to suppress appetite) but in general illicit substances and cannabis and opiates in particular [8]. Similarly, Piran and Robinson [9] reported a co-occurrence of binge eating behaviors with binge alcohol consumption (but not alcohol consumption frequency), cocaine use, and marijuana use in non-clinical university and community samples of women. Like SUD diagnosis studies, drug use was mostly associated with diagnosis of bulimia nervosa, not anorexia nervosa [8]. The associations appear particularly evident in binge eating disorder (BED) which shares characteristics with SUD (including binge drinking), notably impaired impulse control [10]. Grilo et al. [11] reported that $24.8 \%$ of women and men diagnosed with BED in their sample had comorbidity with SUD and that comorbidity was associated with higher report of binge eating episodes (compared to those with BED alone). While BED is associated with obesity, not all obese persons can be diagnosed with BED (see [12]). It is difficult to assess the relationship between SUD and body weight from these studies since few offer measures of body weight. Furthermore, many do not provide information regarding any link between ED and SUD or drug use in men.

\section{Substance Use and Body Weight}

Both cross-sectional and prospective studies suggest that drug use may be "protective" of body weight during active use. Chronic moderate alcohol use is associated with normal or low body mass index (BMI) [13] (see [14] for review). Indeed, alcohol consumption may be protective of body weight in all except the severely obese (BMI $>55)$ where higher alcohol consumption was associated with higher BMI [15]. Some [14] have suggested that binge drinking (in the absence of alcohol dependence) may be more likely linked to elevated body weight but any association may be somewhat dependent on the presence of binge-related eating disorders as well [16]. Furthermore, there was an inverse linear relationship between cannabis use and BMI in a sample of women [17] despite its reputation for elevating appetite. In a sample of those who were in treatment for SUD, the lowest BMIs were found in persons with the heaviest general drug use [18].

Prospective studies have shown similar results when examining weight in relation to risk for SUD diagnosis (but often not frequency of drug use). Adolescent drug use may be protective against weight gain. No significant relationship between drug use as a youth and subsequent body weight or body fat was found over the 2 years examined [19]. Indeed, drug use may be associated with elevated risk of being underweight [19]. There are some reported gender and BMI differences. A diagnosis of SUD was associated with lower BMI in men but was not associated with BMI in women [20]. In an examination of lifetime likelihood and past year SUD risk, overweight men had higher rates of alcohol dependence but overweight women had significantly lower occurrence of alcohol dependence [13]. There were no associations between opiate, marijuana, and cocaine dependence and BMI in men or women [13]. All of these studies examine diagnostic category occurrence and do not examine frequency of drug use or food consumption. However, they do suggest differences in patterns of excess weight by gender in relation to abuse of alcohol (but not other drugs).

Studies of nonclinical populations are less common but their findings are similar to those reported above. In a large sample of young men undergoing evaluation for fitness for national service in Austria, no relationship was found between BMI and alcohol abuse/dependency [21]. Additionally, there was an inverse relationship between BMI and prevalence of illicit drug use [21]. No measures of drug use frequency, drug amounts drugs used, or appetite/eating behavior were made. Similarly, no relationship was found between BMI and "recreational drug use" (while controlling for alcohol and tobacco use, age, levels of sedentary and active behavior, and sleep duration) in a national sample of 72,000 adolescents in Korea [22]. However, in that sample only $1.4 \%$ of participants admitted ever having used drugs while $18.4 \%$ reported BMI in the overweight and obese range (only $13.4 \%$ of girls). In the only study of actual eating behavior in non-SUD persons, those who reported more frequent drug use ate more in a laboratory snack [23•]. In addition, a positive relationship was found between BMI and alcohol use frequency in that sample of 62 university students. In a larger sample of 212 university students, frequency of cannabis use was associated with elevated BMI in women but not in men (unpublished data; method described in [24]). Sex differences have been reported elsewhere. In adolescent girls, higher drug use (cannabis, alcohol, tobacco) was associated with elevated BMI but no relationship between drug use frequency and BMI was found in boys [25]. 


\section{SUD Recovery and Body Weight}

While an association between body weight and active drug use is weak, there is evidence that patients in recovery from SUD may be at risk for weight gain. In their sample of abstinent patients diagnosed with substance abuse, Jackson and Grilo [26] reported that $65 \%$ were overweight (of whom $20 \%$ were obese). Sixty percent reported weight fluctuations of $9 \mathrm{~kg}$ or more. However, their patients were not examined for changes in weight during the treatment period and were not compared to control participants. Evidence for changes in BMI during treatment is present in the majority of studies which examined body weight during recovery from SUD. Most of the research has focused on patients in treatment for opiates.

The association of smoking cessation with weight gain is reviewed elsewhere [27] and is frequently assumed to be primarily a rebound from the appetite suppressing effects of nicotine. Smoking is often thought to be anorectic and smokers do have lower body weight despite eating the same or more as nonsmokers [28]. However, smoking may enhance the reinforcement provided by food (perhaps due to alteration of phasic dopamine release associated with reinforcement) and this enhancement may persist after smoking cessation [28]. Thus, the weight gain observed in smokers who quit may be more than a rebound; there may be an increased incentive for food in recovering smokers [28].

Heroin detoxification and opiate replacement therapies are frequently associated with weight gain. One of the earliest studies including a measure of body weight reported that $40 \%$ of those on methadone experienced weight gain [29]. More recent studies have reported similar results. Both men and women in treatment for opiate dependency gained weight $(5.3 \%$ increase, mean $=4.5 \mathrm{~kg}$ and $7.5 \%$ increase, mean $=5.0 \mathrm{~kg}$ respectively) during a 60 -day buprenorphine (or methadone) treatment period [29]. Mysels et al. [30] reported a small increase in weight gain for patients in methadone $(3.7 \%)$ and naltrexone treatments $(6.7 \%)$ over a 6 month period which amounted to an approximate $4.5 \mathrm{~kg}$ increase. Weight gain was not significantly different between the treatments.

Patterns of BMI increase have been examined in longer term studies as well. Reimer et al. [31] reported only very small (but significant due to large sample size) increases in BMI (1 BMI point) over one year for patients in methadone and heroin treatments. The mean BMIs remained in the normal range and were not higher than that of participants who dropped out of the treatment. Kolarzyk et al. [32] reported that women $(n=7)$ on methadone after 4 years had slightly lower weight $(1.7 \mathrm{~kg})$ and no change in body fat compared with admission to study but that men $(n=23)$ had elevated weight $(8.8 \mathrm{~kg})$ and an increase in body fat (from $11.9 \%$ to $18.5 \%$ ) compared to admission to the study. Eight of the men moved up in BMI classification. Okruhlika \& Slezáková [33] measured weight annually over a 4 year period in a group of male and female methadonetreated patients $(\mathrm{N}=42)$. They reported significant increases in weight gain in the first $(7.5 \mathrm{~kg})$ and second $(2.5 \mathrm{~kg})$ year which remained stable for the rest of the study period. The portion of the sample which moved into overweight ( $8 \%$ to $33 \%)$ and obese ( $2 \%$ to $17 \%$ ) classifications was also significant although the mean BMI was not deemed different than what would be expected in the population [33]. In a cross-sectional study of weight and opiate treatment [34], male and female outpatients on methadone maintenance (mean BMI $=28.85$ ) weighed significantly more than agematched controls (mean BMI $=22.84$ ). The weight of the patients was unrelated to length of methadone treatment. Not all reports of BMI change have indicated an increase: Morabia et al. [35] reported that men on methadone treatment had $5.3 \%$ lower BMI than controls (with no difference for women) and that mean BMI for all was within the normal range. Their data suggest that their patients replaced foods rich in fats and animal proteins with those rich in carbohydrates so that their total energy consumption was not different. Since this is a cross sectional study, it is not known whether their weight at the time of measure was different than when they entered treatment.

In a qualitative study [36], men studied in three stages of recovery (mostly from cocaine use) reported excess weight gain (between 6.4 and $20.4 \mathrm{~kg}$ over the first 6 months of recovery), using food as substitute for drug or as mood regulator, and disordered eating (primarily bingeing). Six men in mid-recovery (7-13 months) reported gaining 15 to $27.2 \mathrm{~kg}$ in early treatment but three of them reported losing 0.5 to $6.8 \mathrm{~kg}$ by restricting food consumption by mid treatment. Similar patterns of early weight gain were reported retrospectively by those in late recovery [36]. Male alcoholics in treatment gained significantly more weight $(3.4 \mathrm{~kg})$ than controls $(0.2 \mathrm{~kg})$ over a 6 month period [37]. Adolescents in residential drug treatment gained weight in the first 60 days observed ( $5 \mathrm{~kg}$ on average) with less gained later on [38]. The percent overweight increased from $5.8 \%$ to $13.5 \%$. In a rare weight intervention study, weight gain in SUD recovery was prevented. Male adolescents in inpatient treatment were randomly assigned to either exercise or exercise/nutrition counseling and compared to untreated past "population" [39]. The combined treatment group had lower BMI than control and past patients without the 5060 day weight loss intervention.

In summary, studies of patients in SUD recovery describe an increase in body weight between approximately 3.4 and $27.2 \mathrm{~kg}$. Those that have measured body weight over time allow monthly weight gain to be estimated at between 0.5 to $4.5 \mathrm{~kg}$ per month relatively early in recovery. Not all investigations have demonstrated a change in BMI during SUD 
recovery. Zador et al. [40] found no difference in BMI in a sample of 86 women on methadone maintenance when compared to national population data despite reports of significantly higher sucrose consumption. BoguckaBonikowska et al. [41] reported no difference in BMI between men on methadone maintenance $(\mathrm{N}=30)$ and controls $(\mathrm{N}=32)$. No BMI difference was reported between abstinent alcoholics and controls [42, 43]. In short and long term longitudinal studies, weight gain has been reported in persons ranging in age from adolescence to middle age, in men and women (although more data are available from men), in the US and Europe, and when using self-report and measured body weight. Cross sectional studies (especially of alcohol dependent men) are more likely to report no difference in body weight between patient and control groups. This discrepancy may be due to significant individual differences in weight change.

\section{SUD Recovery and Eating Behavior and Taste}

Little is known about the eating behavior of patients in SUD recovery and what is known comes exclusively from dietary recall and taste tests. Some evidence has emerged that SUD recovery is associated with changes in eating and food preference: particularly in regard to sweets. Changes in consumption associated with taste may reflect an enhancement of food-derived pleasure or of food-related cue salience. Some of these differences in eating and taste occur with weight changes while in some cases they do not (or no weight information is provided in the study).

Elevated preference for sweets and/or increased sugar consumption has been commonly reported in persons in recovery from opiate dependence. Gambera and Clarke [29] reported that nearly $90 \%$ of their sample of men and women experienced craving for sweets while in heroin detoxification and $60 \%$ reported it while on methadone maintenance. Sixty percent reported having higher appetite or eating more often despite a nutritionally inadequate diet [29]. Some reports of sex differences appear in this literature. Over a 4-year study, men on methadone maintenance did not recall higher energy consumption while women reported a $500 \mathrm{kcal}$ (nonsignificant) increase (only seven women in study) [44] despite a small weight loss. This sample also experienced excessive sweet consumption at the initiation of the study with little change in diet over the treatment period (although there was some improvement in sugar consumption) [45]. In general, diet improved toward the end of test period but not to recommended levels [45]. Cross-sectional studies have also found that male and female methadone-treated patients report eating more sweet foods (especially sugars) than control participants [35, 40]. More recent studies suggest that changes in food preference in methadone patients may not be restricted to sweets. Nolan and Scagnelli [34] reported that methadone patients reported higher consumption of "junk" foods, snacks and sweets than controls as well as a higher degree of weight concern and dieting. When asked what they would do if provided with the foods on a list at the moment of questioning, the patients who wished to eat reported higher desire to consume sweets and savories and larger food portions than age-matched controls [34]. Cocores and Gold [46] reported that men and women described increased consumption of salty "fast food" during recovery from opiate dependency. The mechanism for these changes is unclear although there is evidence linking opioid transmitters to sweet preference [47].

Similar relationships have been found in those recovering from non-opiate substances. Cowan and Devine [36] reported a time course for change in eating behavior in their sample of men recovering from cocaine dependence: food bingeing and hoarding occurred in early phases of recovery (first 6-7 months) with movement toward more normal eating patterns (albeit with larger portions) after about a year. Detoxified male alcoholics reported having a strong desire to eat sweets [48]. Male and female detoxified alcoholics reported a significant $37.1 \%$ increase in consumption of sweets but no difference in BMI was present between patients starting consumption of and those reducing or ending consumption of alternative substances (cigarettes, chocolate, sweets, and coffee) [49]. There was an association between self-reported carbohydrate $(\mathrm{CHO})$ "craving" and alcohol craving in abstaining alcoholic men identified as carbohydrate cravers (50\% of the sample of alcoholics) but not in those without carbohydrate cravings [50]. This occurred even when assigned to a high $\mathrm{CHO}$ diet. No BMI measures were made so an association with body weight could not be determined.

Finally, there are several reports of changes in taste preference in patients recovering from cocaine and alcohol dependency. The only study of preferences in persons $(\mathrm{N}=16)$ with cocaine dependency shows an increased preference for sweet solutions [51]. Similarly, recently detoxified alcoholics rated the intensity and pleasantness of sucrose solutions as very strong more often than control participants and alcoholics 24 days into treatment reported their maximal pleasantness response to the highest sucrose concentration presented when compared to control participants [37, 52, 53]. The maximal pleasant response to the sweetest tastants occurred after 1 month of abstinence and decreased over time [37]. Unfortunately, no measures of BMI or food consumption were made in several of these studies so no relationship with body weight can be determined. Several studies have reported no changes in sweet responsiveness for methadone patients [41] or abstinent alcoholics [42, 43]. 


\section{Conclusion}

Complex relationships exist between body weight and substance use. Research findings suggest that a SUD diagnosis (and possibly drug use itself) may be protective of body weight but that recovery from SUD elevates risk of body weight increase. The mechanism for weight increase is undetermined.

Changes in eating might be a sort of "replacement" for the drug of choice [36]. Junghanns et al. [49] reported that $78.2 \%$ of detoxified alcoholics described an increase in consumption of alternative substances: coffee, cigarettes, chocolates and other sweets. Goldstein et al. [54] reported that active cocaine users rate wanting for food and sex as greater than wanting for drugs in "current lab" and "general" situations but reverse it when "under the influence." Thus, increased consumption of alternate caloric substances might lead to increases in weight. The possible shift from one substance to another and the evidence that drug use is protective of body weight suggests that there is a competition among substances/activities for brain reward sites [17]. Overeating may displace drug use while drug use may displace food consumption (in addition to any anorectic effects the drug itself may have). According to this competition theory, food consumption increases when drugs are no longer being used (or no longer available) to activate reward sites. However, it is unlikely to be purely a substance replacement since, in the case of drug replacement therapies (i.e., methadone), there remains a pharmacological agent present in the brain.

It is possible that drug abstinence produces changes in the pleasantness of sweet stimuli (and other taste stimuli) which might alter the consumption of sweet foods or sugars in particular. However, the role of pleasantness in compulsive drug and food consumption is controversial. The dysfunction in dopamine systems associated with reinforcement is probably not mediating excessive or deficient pleasure derived from these activities. It is commonly mistakenly assumed that "reward" means pleasure but in most studies reward appears to be used (erroneously) as a synonym for reinforcement [1]. Evidence suggests that central dopamine systems are more involved in "wanting" [55]. While sensitization of incentives ("wanting") in one domain (i.e., drugs) does not appear to make all rewards equally wanted, there may be cross sensitization from one enhanced reward to another [56], for example, palatable food. "Wanting" may be more important than "liking" in the interaction between food consumption and drug use in humans. In the only study of actual eating behavior in relation to drug use, Nolan and Stolze [23•] found that the association between elevated drug use and higher food consumption was mediated by desire to consume the food present in mouth and not pleasantness of the food being consumed.
It is also possible that weight increases in those in SUD recovery are due to a rebound in body weight from abnormally low weights associated with drug use. Indeed there are reports that persons with active SUD are poorly nourished. Best et al. [57] reported low rates of meal consumption among patients $(\mathrm{N}=100)$ on methadone maintenance $(63 \%$ ate on less than six occasions over the 3 days prior to testing). These patients also reported high rates of smoking and alcohol consumption. Those who ate more meals drank less alcohol; alcohol appeared to be predictive of nutritional state (not methadone or smoking) [57]. Unfortunately, no report of BMI or rates of overweight were included. A more recent report suggests that many within early phases of SUD recovery are normal weight. While some patients in detoxification are poorly nourished, $76 \%$ of those who completed 4 -week detoxification regime $(\mathrm{N}=59)$ were well nourished (despite micronutrient deficits) [58]. No relationship was found between nutrition status and drug of choice and better energy intake predicted higher BMI [58]. Those with poor nutrition status were in normal range for BMI while those assessed as well nourished were overweight [58]. Cowan and Devine [36] suggest that the weight gain they observed in men recovering from SUD exceeds that which would be necessary to bring body weight to normal. However, they do note that the SUD recovery period shares some of the eating characteristics (such as food hoarding) of food-deprived men returned to normal feeding regime [59]. The time course of behavior, craving and weight gain is also similar [59].

Drug use and eating may interact indirectly through other behavioral systems. It is unclear whether changes in body weight and taste preferences associated with substance use are due to the use of drugs and alcohol, whether the substance use is due to pre-existing taste preferences and eating behavior, or whether both are manifestations of a common behavioral tendency such as impulsivity, risk taking, or sensation seeking [23•]. Evidence suggests that substance use increases impulsivity and vice versa, as well as the possibility that a third factor such as reactivity to non-drug rewards such as food (among other factors) may underlie the relationship [60]. Reward sensitivity has been suggested to be a critical variable in the development of substance use and eating disorders [10] and has been implicated in higher food-cue reactivity [61]. Any changes in eating behavior and/or body weight may be the result of underlying tendencies to impulsivity and/or reactivity that manifest themselves not only in higher rates of substance use but higher consumption of food [23•].

The few studies of drug use which examine BMI suggest small increases in body weight. Fewer examine frequency of drug use, body composition, appetite, and eating behavior making it difficult to assess the role of eating behavior in any substance use-related weight gain. While evidence is consistent with theories of sensitized incentives, more data need to be 
collected. The relationships known suggest that patients in treatment for substance use may benefit from nutritional and weight management counseling. Furthermore, the obese may benefit from techniques known to be effective in controlling cravings and/or impulsivity among those in treatment for substance dependence.

Disclosure No potential conflicts of interest relevant to this article were reported.

\section{References}

Papers of particular interest, published recently, have been highlighted as:

- Of importance

1. Salamone JD, Correa M. Dopamine and food addiction: lexicon badly needed. Biol Psychiatry. 2013, in press.

2. - Volkow ND, Wang GJ, Fowler JS, et al. Food and drug reward: overlapping circuits in human obesity and addiction. Curr Topics Behav Neurosci. 2012;11:1-24. This is a thorough review of the recent research using neuroimaging to examine the similarities in brain reinforcement center dysfunction in both obesity and substance dependence. They propose that the discrepancy between anticipation for the food/drug effects and the blunted neurophysiological effects of them maintain the taking of drugs/overconsumption of food in an attempt to achieve the expected reinforcement.

3. Avena NM, Wang M, Gold MS. Implications of food addiction and drug use in obesity. Psychiatr Ann. 2011;41:478-82.

4. Smith DG, Robbins TW. The neurobiological underpinnings of obesity and binge eating: a rationale for adopting the food addiction model. Biol Psychiatry. 2013, In press.

5. - Ziauddeen H, Farooqi IS, Fletcher PC. Obesity and the brain: how convincing is the addiction model? Nat Rev Neurosci. 2012;13:27986. The authors of this paper thoroughly examine the criteria used for diagnosis of substance use disorders and whether they are applicable to the proposed diagnostic scheme for food addiction. They conclude that there is a tendency to see all of the evidence on neural and behavioral similarities as supportive of a food addiction model for obesity despite significant inconsistencies.

6. Holderness CC, Brooks-Gunn J, Warren MP. Co-morbidity of eating disorders and substance abuse: review of the literature. Int J Eat Disord. 1994;16:1-34.

7. Nøkleby H. Comorbid drug use and eating disorders - a review of prevalence studies. Nordic Stud Alcohol Drugs. 2012;29:303-13.

8. Calero-Elvira A, Krug I, Davis K, et al. Meta-analysis on drugs in people with eating disorders. Eur Eat Disord Rev. 2009;17:243-59.

9. Piran N, Robinson SR. Patterns of associations between eating disordered behaviors and substance use in two non-clinical samples: a university and a community based sample. J Health Psychol. 2011;16:1027-37.

10. Dawe S, Loxton NJ. The role of impulsivity in the development of substance use and eating disorders. Neurosci Biobehav Rev. 2004;28:343-51.
11. Grilo CM, White MA, Masheb RM. DSM-IV psychiatric disorder comorbidity and its correlates with binge eating behavior. Int J Eat Disord. 2009;42:228-34.

12. Davis C. Psychobiological traits in the risk profile for overeating and weight gain. Int J Obes (Lond). 2009;33:549-53.

13. Barry D, Petry NM. Associations between body mass index and substance use disorders differ by gender: results from the National Epidemiologic Survey on Alcohol and Related Conditions. Addict Behav. 2009;34:51-60.

14. Yeomans MR. Alcohol, appetite and energy balance: is alcohol intake a risk factor for obesity? Physiol Behav. 2010;100:82-9.

15. Kleiner KD, Gold MS, Frost-Pineda K, et al. Body mass index and alcohol use. J Addict Dis. 2004;23:105-18.

16. Eichen DM, Conner BT, Daly BP, et al. Weight perception, substance use, and disordered eating behaviors: comparing normal weight and overweight high-school students. J Youth Adolesc. 2012;41:1-13.

17. Warren M, Frost-Pineda K, Gold M. Body mass index and marijuana use. J Addict Dis. 2005;24:95-100.

18. Cofrancesco J, Brown TT, Luo RF, et al. Body composition, gender, and illicit drug use in an urban cohort. Am J Drug Alcohol Abuse. 2007;33:467-74.

19. Pasch KE, Velazquez CE, Duncan Cance J, et al. Youth substance use and body composition: does risk in one area predict risk in the other? J Youth Adolesc. 2012;41:14-26.

20. Pickering RP, Goldstein RB, Hasin DS, et al. Temporal relationships between overweight and obesity and DSM-IV substance use, mood, and anxiety disorders: results from a prospective study, the National Epidemiologic Survey on Alcohol and Related Conditions. J Clin Psychiatry. 2011;72:1494-502.

21. Blüml V, Kapusta N, Vyssoki B, et al. Relationship between substance use and body mass index in young males. Am J Addict. 2011;21:72-7.

22. Baek S-I, So W-Y. Association between weight status and recreational drug abuse in Korean adolescents. Cent Eur J Med. 2012;7:224-9.

23. - Nolan LJ, Stoltz MR. Drug use is associated with elevated food consumption in college students. Appetite. 2012;58:898-906. This is the first study to examine whether drug use predicts actual food consumption. They found that drug use predicted higher consumption of several of the snacks provided and elevated appetite ratings. The increase in consumption was moderated by elevated desire to consume food and not the pleasantness of the food. Thus, drug use may influence food consumption in students who have not been diagnosed with substance use disorders.

24. Nolan LJ. Association between impulsiveness and pleasantness ratings for food and drugs. Appetite. 2012;59:e43.

25. Farhat T, Iannotti RJ, Simons-Morton BG. Overweight, obesity, youth, and health-risk behaviors. Am J Prev Med. 2010;38:258-67.

26. Jackson TD, Grilo CM. Weight and eating concerns in outpatient men and women being treated for substance abuse. Eat Weight Disord. 2002;7:276-83.

27. Filozof C, Fernández Pinilla MC, Fernández-Cruz A. Smoking cessation and weight gain. Obes Rev. 2004;5:95-103.

28. Donny EC, Caggiula AR, Weaver MT, et al. The reinforcementenhancing effects of nicotine: implications for the relationship between smoking, eating, and weight. Physiol Behav. 2011;104:143-8.

29. Gambera SE, Clarke JK. Comments on dietary intake of drugdependent persons. J Am Diet Assoc. 1976;68:155-7.

30. Mysels DJ, Vosburg S, Benga I, et al. Course of weight change during naltrexone vs. methadone maintenance for opioiddependent patients. J Opioid Manag. 2011;7:47-53.

31. Reimer J, Verthein U, Karow A, et al. Physical and mental health in severe opioid-dependent patients within a randomized controlled maintenance treatment trial. Addiction. 2011;106:1647-55. 
32. Kolarzyk E, Wojtowicz B, Szpanowska-Wohn A, et al. Nutritional status of the opiate dependent persons after 4 years of methadone maintenance treatment. Przegl Lek. 2005;62:373-7.

33. Okruhkica L', Slezáková S. Weight changes of patients in methadone maintenance treatment during four years period. Cas Lek Cesk. 2012;151:389-91.

34. Nolan LJ, Scagnelli LM. Preference for sweet foods and higher body mass index in patients being treated in long-term methadone maintenance. Subst Use Misuse. 2007;42:1555-66.

35. Morabia A, Fabre J, Chee E, et al. Diet and opiate addiction: a quantitative assessment of the diet of non-institutionalized opiate addicts. Br J Addict. 1989;84:173-80.

36. Cowan J, Devine C. Food, eating, and weight concerns of men in recovery from substance addiction. Appetite. 2008;50:33-42.

37. Krahn D, Grossman J, Henk H, et al. Sweet intake, sweet-liking, urges to eat, and weight change: relationship to alcohol dependence and abstinence. Addict Behav. 2006;31:622-31.

38. Hodgkins C, Cahill KS, Seraphine AE, et al. Adolescent drug addiction treatment and weight gain. J Addict Dis. 2004;23:55-65.

39. Hodgkins C, Frost-Pineda K, Gold MS. Weight gain during substance abuse treatment. J Addict Dis. 2007;26(S1):41-50.

40. Zador D, Lyons Wall PM, Webster I. High sugar intake in a group of women on methadone maintenance in South Western Sydney, Australia. Addiction. 1996;91:1053-61.

41. Bogucka-Bonikowska A, Baran-Furga H, Chmielewska K, et al. Taste function in methadone-maintained opioid-dependent men. Drug Alcohol Depend. 2002;68:113-7.

42. Bogucka-Bonikowska A, Scinska A, Koros E, et al. Taste responses in alcoholic men. Alcohol Alcohol. 2001;36:516-9.

43. Wronski M, Skrok-Wolska D, Samochowiec J, et al. Perceived intensity and pleasantness of sucrose taste in male alcoholics. Alcohol Alcohol. 2007;42:75-9.

44. Kolarzyk E, Chrostek Maj J, Pach D, et al. Assessment of daily nutrition ratios of opiate-dependent persons before and after 4 years of methadone maintenance treatment. Przegl Lek. 2005;62:368-72.

45. Kolarzyk E, Jenner B, Szpanowska-Wohn A, et al. The changes in taste preferences during 4 years period of methadone maintenance treatment. Przegl Lek. 2005;62:378-81.

46. Cocores JA, Gold MS. The salted food addiction hypothesis may explain overeating and the obesity epidemic. Med Hypotheses. 2009;73:892-9.
47. Drewnowski A, Krahn DD, Demitrack KN, et al. Taste responses and preferences for sweet high-fat foods: evidence for opioid involvement. Physiol Behav. 1992;51:371-9.

48. Junghanns K, Backhaus J, Tietz U, et al. The consumption of cigarettes, coffee and sweets in detoxified alcoholics and its association with relapse and family history of alcoholism. Eur Psychiatry. 2005;20:451-5.

49. Junghanns K, Veltrup C, Wetterling T. Craving shift in chronic alcoholics. Eur Addict Res. 2000;6:64-70.

50. Moorhouse M, Loh E, Lockett D, et al. Carbohydrate craving by alcohol-dependent men during sobriety: relationship to nutrition and serotonergic function. Alcohol Clin Exp Res. 2000;24:635-43.

51. Janowsky DS, Pucilowski O, Buyinza M. Preference for higher sucrose concentrations in cocaine abusing-dependent patients. J Psychiatr Res. 2003;37:35-41.

52. Kampov-Polevoy AB, Tsoi MV, Zvartau EE, et al. Sweet liking and family history of alcoholism in hospitalized alcoholic and nonalcoholic patients. Alcohol Alcohol. 2001;36:165-70.

53. Kampov-Polevoy AB, Eick C, Boland G, et al. Sweet liking, novelty seeking, and gender predict alcoholic status. Alcohol Clin Exp Res. 2004;28:1291-8.

54. Goldstein RZ, Woicik PA, Moeller SJ, et al. Liking and wanting of drug and non-drug rewards in active cocaine users: the STRAP-R questionnaire. J Psychopharm. 2010;24:257-66.

55. Berridge KC, Ho C-Y, Richard JM, et al. The tempted brain eats: pleasure and desire circuits in obesity and eating disorders. Brain Res. 2010;1359:43-64.

56. Berridge KC. The debate over dopamine's role in reward. The case for incentive salience. Psychopharmacology. 2007;191:391-431.

57. Best D, Lehmann P, Gossop M, et al. Eating too little, smoking and drinking too much: wider lifestyle problems among methadone maintenance patients. Addict Res. 1998;6:489-98.

58. Ross LJ, Wilson M, Banks M, et al. Prevalence of malnutrition and nutritional risk factors in patients undergoing alcohol and drug treatment. Nutrition. 2012;28:738-43.

59. Keys A, Brozek J, Henschel A, et al. The biology of human starvation, vol. 2. Minneapolis: University of Minnesota Press; 1950.

60. Perry JL, Carroll ME. The role of impulsive behavior in drug abuse. Psychopharmacology. 2008;200:1-26.

61. Tetley AC, Brunstrom JM, Griffiths PL. The role of sensitivity to reward and impulsivity in food-cue reactivity. Eat Behav. 2010;11:138-43. 